\title{
Planning sustainable community health schemes in rural areas of developing countries
}

\author{
Honora K. Smith ${ }^{a *}$, Paul R. Harper ${ }^{b}$, Chris N. Potts ${ }^{a}$, \\ Ann Thyle ${ }^{b}$ \\ ${ }^{a}$ School of Mathematics, University of Southampton, \\ Southampton, SO17 1BJ, UK \\ ${ }^{b}$ Cardiff School of Mathematics, Cardiff University, \\ Senghennydd Road, Cardiff, CF24 $4 A G, U K$ \\ ${ }^{c}$ Emmanuel Hospital Association, 808/92 Nehru Place, \\ New Delhi - 110019, India
}

${ }^{*}$ Corresponding author. Tel: +44238059 3647; fax: +44238059 2225; e-mail: hs@maths.soton.ac.uk 


\begin{abstract}
In this research, we consider the planning of community health schemes by non-governmental or faith-based organisations in rural areas of developing countries, from both top-down and ground level viewpoints. We conclude that both types of planning approach are valid and necessary for sustainability of such developments. With top-down planning in mind, we describe our hierarchical models especially designed for location of community health facilities, with objectives pertaining to both efficiency and equity of provision. As an additional case study, we present modelling of the location of a maximal number of self-sustainable primary healthcare workers in a rural region of India.
\end{abstract}

Keywords: OR in developing countries; Location; OR in health services 


\section{Introduction}

A high percentage of the world's poor live in rural areas of developing countries where access to health care is almost completely lacking. Poor health may cause entire families to drop into abject poverty, because of inability to work or the necessity to sell assets in order to pay for treatment (Barr, 2002). Government provision often fails to reach those who should receive it and private health care is available in general only to the wealthy living in cities and large towns (Preker et al., 2002). It is often only non-governmental organisations (NGOs) and faith-based organisations (FBOs) that are motivated to bridge the gap with community health schemes, albeit on a localised scale. Such schemes, whether focusing on medical or social development, are often funded for a fixed time period only, without planning for sustainability. FBOs, however, are particularly motivated to provide the long-lasting benefit to local communities that sustainability brings. In this research, we seek to improve the planning process of such community schemes.

We consider community health and development schemes in rural areas of developing countries. In such areas, it is often considered appropriate to instigate development work such as literacy teaching and self-help groups alongside health projects, because of prevailing low standards of education and social development. Improvements in health and social conditions are concomitant as development enables communities to escape from the trap of poverty. Sustainability of such integrated schemes may depend on a number of factors: financing mechanisms vary, as do local conditions which determine the efficiency of operation. Local participation in a scheme changes attitudes from dependency to active involvement. Furthermore, the growth of trust in a health provider affects the use made of health facilities, leading to sustainability of any particular project. 
Our research is based on experience of community health and development projects administered by Emmanuel Hospital Association (EHA) in northern India. We consider top-down, overall approaches to planning for sustainability as well as ground-level considerations. We conclude that both approaches are valid and necessary for the planning of sustainable development. As a contribution to overall planning, we propose location models specifically designed for healthcare situations in rural areas of developing countries. We bear in mind the possible objectives of FBOs in making such provision: this gives our modelling its unique character.

A variety of objectives may be appropriate to the provision of healthcare facilities in a rural developing area. In particular, there may be differences in the nature of hierarchical healthcare provision, from village to hospital level. For example, one provider might be concerned that everyone living in a target area should have ready access to facilities at every level from hospital to village level. Another healthcare provider in a different region might aim to cover those in remote parts with a referral system, providing extra transport where necessary to high level facilities. Furthermore, an FBO might be concerned that those living below the poverty line should have equitable access to health care. We therefore propose a range of hierarchical models for planning sustainable community health schemes. In these models, we reflect differences in access available to hierarchical facilities, with objectives relating to concerns for both equity and efficiency of provision.

In addition, we consider as a case study the REACH scheme (Morse, 2006), for which a pilot project is proposed in the Karur District of Tamil Nadu, South India. This scheme for primary healthcare provision is of particular interest in terms of sustainability. It has been designed to take advantage of existing self-help groups, through which the services of health care workers (HCWs) may be channelled. A sufficient density of self-help groups is needed for sustainable employment of the HCWs. Our model for 
location of the maximal number of sustainable facilities (MNS)

demonstrates possible solutions to this problem.

Contacts have been made with a number of health professionals working in a variety of community health schemes in developing countries. We are encouraged by the response that our approach is valid in terms of potential usefulness.

The paper progresses as follows. Firstly, in Section 2, we review some of the OR approaches made to planning community healthcare and social development. In Section 3, we give a general description of community health schemes in rural areas of developing countries, with specific details of EHA projects and of planning for sustainability from the ground-level point of view. We describe in Section 4 our hierarchical location models developed for overall planning of rural healthcare facilities, with options for both efficient and equitable objectives. As a case study, we introduce the MNS model in Section 5, applied to the REACH scheme (Morse, 2006) for primary healthcare provision. We give our conclusions in Section 6, along with directions for future work.

\section{OR approaches to community healthcare and social development planning}

Mingers and Rosenhead (2001) highlight the parallels between development planning approaches and OR methods. In both arenas, there has been a change from 'hard' analytical methods to 'soft' participative approaches. In OR approaches to planning for development itself, we therefore expect to see such changes in methods.

Firstly, we give examples of 'hard' OR techniques applied to planning for development. Use of $p$-median (Hakimi, 1964, 1965) and set-covering 
models (Toregas et al., 1971) are suggested by Banerji and Fisher (1974) for planning in rural India, with an application in the Guntur district of Andhra Pradesh. Patel (1979) describes an analysis carried out to determine optimal locations for service centres in Dharampur, a poor rural area in Gujarat, India. Competing interests sought locations in either hilly or planar regions: modelling according to set-covering techniques (Toregas et al., 1971) helped to settle disputes, and successful implementation was achieved. A maximal covering location model (Church and ReVelle, 1974) is used by Eaton et al. (1981) to improve upon planners' siting of ambulance bases and centres from which to recruit rural health workers. A number of recommendations are implemented, and decision-makers have taken up the modelling techniques elsewhere in Colombia. Rushton (1984) gives a review of use made of location-allocation models in planning services in rural areas of developing countries. A hierarchical location model is applied by Hodgson (1988) to primary healthcare delivery in the Salcette region of Goa, India, while Hodgson et al. (1998) use a covering tour model to plan mobile health services in the same district. The length of tour is minimised, subject to the constraint that the tour should be less than a certain minimum distance from village centres not visited.

More recently, Rahman and Smith (2000) review the potential usefulness of location-allocation models in planning health services in developing countries. A number of studies are considered, of both single-level and hierarchical systems. The links between healthcare and economic development are emphasised. The frequent absence of implementation of solutions is thought to be caused by lack of local involvement and communication with decision makers. Yasenovskiy and Hodgson (2007) consider the realism of representation of spatial hierarchies of facilities. Several location-allocation models are compared, which increase in realistic representation: the hierarchical $p$-median model, a spatial interaction-based hierarchical model, and a new spatial choice-based hierarchical model. The 
$p$-median model is seen to be unrealistic, as clients frequently bypass lower level facilities for larger service centres. Spatial interaction assumes that demand is proportional to facility attractiveness and inversely proportional to the disutility of travel. The spatial choice-based model additionally incorporates choices between destinations. Application is made to data from healthcare facilities in Suhum District, Ghana.

'Soft' OR methods have been applied to development issues in recent years with successful outcomes. Mingers and Rosenhead (2001) describe and review the use of Community Operational Research with disadvantaged groups; use of problem structuring methods is reported, in Britain and elsewhere. In the UK, Jackson (2006) promotes the use of Community Operational Research in an evaluation of a Healthy Living Centre in Lincolnshire. Use of rapid appraisal techniques is described by Lewis et al. (2003) in community engagement in Hillbrow, South Africa.

\section{Community health projects in developing countries: ground level conditions for sustainability}

This section further characterises community health schemes, giving specific details of EHA projects. We consider aspects of the sustainability of schemes from ground-level viewpoints. Recommendations for best practice are taken from Lankester (2000).

\subsection{General features of community healthcare schemes}

Community projects offering aspects of health care and development assistance are commonly initiated by NGOs or FBOs in rural regions of developing countries, where residents have very limited access to quality medical care. 
Community healthcare schemes may be set up as an extra activity by a hospital, to reach out to people living in inaccessible parts. Alternatively, schemes may run independently, but have links to one or more hospitals for the purpose of referral of seriously-ill patients. Projects may be targeted on treatment of particular diseases such as eye defects, TB or HIV/AIDS, or may be more generally orientated. Maternity care and child health is often a concern, as is evidenced by the Millennium Development Goals (UN, 2002, 2006).

\subsection{EHA community health and development projects in northern India}

Our research is focused on community health projects in northern India administered by EHA, an indigenous Indian healthcare provider. EHA operates at village, community and hospital level, with the objective of providing affordable health services to the poorest people in rural communities, in an equitable manner. Alongside medical interventions, community development activities aim to empower participants in making health-enhancing choices. Improvements in the maternal mortality rate and infant mortality rate are of particular interest.

EHA operates a number of community health and social development schemes across northern India. These projects take a variety of forms, for example:

- Ante-natal care and child immunisation

- TB treatment

- Women's self-help groups

- Adolescent groups

- Literacy classes 
- Village telemedicine

Data have been collected from EHA community health and development projects in the states of Uttaranchal, in districts near Herbertpur Christian Hospital, and in Uttar Pradesh. Baseline surveys had been carried out before the community schemes commenced. These house-to-house surveys had been undertaken in all villages of the target areas over a period of six months. Data collected include poverty levels, living conditions, transport available and access made to health facilities. In addition, hand-written records have been made available concerning the operation of the community schemes. We make use of these data in modelling the location of community health schemes for both efficiency and equity.

Many EHA community schemes depend on aid from donor organisations which is guaranteed for a limited term only. However, a reproductive and child health $(\mathrm{RCH})$ centre was initiated by Prem Sewa Hospital in Utraula, Uttar Pradesh, with the goal of self-sustainability within two years. Baseline surveys had been carried out prior to commencement of earlier educational development projects. Data regarding demand for services at the $\mathrm{RCH}$ centre will be used in a second phase of our research into the dynamic growth of demand for community health facilities over a geographical region.

\subsection{Sustainability at ground-level: social development}

We believe that the encouragement of social development is an important factor in ensuring sustainability of community health schemes, as discussed in Section 1. We illustrate this belief in considering some of the types of projects mentioned in Section 3.2. For example, women's self-help groups might be established. We describe the economic activities of these groups in Section 5. As income is generated, family nutrition can be improved and better choices made for health, such as making use of a healthcare facility. 
Development activities such as literacy classes can empower the local population with the ability to make vital choices for improvement of conditions. In addition, trust can grow in a new health provider, as familiarity grows with services offered. For example, an adolescent class designed for healthcare teaching and advice can bring greater readiness to seeking ante-natal care in following years.

\subsection{Sustainability at ground-level: recommendations for best practice from Lankester (2000)}

We detail here recommendations made by Lankester (2000) to ensure successful operation of community health and development projects. We consider such ground-level planning considerations to be of importance to sustainability.

Lankester (2000) gives practical details of best practice for effective community-based health schemes, basing methods on the belief that success of community projects is allied with successful training of people at all levels in health care and prevention of disease. Not just those with medical qualifications, but community representatives and family carers make an important contribution to health improvement. In similar vein, the World Health Organization (WHO, 2002) blueprints an initiative for local care of the chronically sick. EHA claims that " $80 \%$ of illness in North India can be prevented or treated at village or slum level without the need of a doctor or nurse" (EHA, 2006). Källander et al. (2006) demonstrate the ability of community health workers, i.e. local residents with basic training, to recognise pneumonia.

The views of Lankester (2000) are derived from first-hand experience gained in directing projects in Himalayan villages. Partnership with the local community is seen to be of paramount importance; active participation rather than passive dependence is to be encouraged by the 
healthcare provider. Initially, methods of getting to know the community similar to Rapid Rural Appraisal or Participative Rural Appraisal (Mingers and Rosenhead, 2001) are recommended, in advance of carrying out baseline surveys. (These methods form a part of the body of participative planning approaches discussed in Section 2).

Importance is given by Lankester (2000) to those working closest to village populations in community health schemes. At village level, residents chosen by the community can be trained as village health workers. Such workers can dispense simple medicines, including antibiotics, and give healthcare training, provided they are sufficiently well informed to know when to refer to more expert sources of help. At community level, workers with extra training can help to staff a community health centre or clinic, offering services such as ante-natal care, child health clinics and blood tests, under the supervision of a nurse or doctor. Again, referral to a hospital should be available for the seriously ill.

Regarding financing of local health schemes, Lankester (2000) suggests that sustainable support for village health workers may be obtained through affordable payments, such as payments in kind, from those who receive their services. The importance can thus be seen of the appointment by communities of their own health workers, as workers are effectively supported while they give time that would otherwise be spent in subsistence farming for survival. Likewise, clinic services should be charged at affordable rates, to ensure sustainability.

\section{Overall planning: locational analysis applied to hierarchical community health schemes}

In seeking improved planning of community health schemes, we investigate the use of location theory for optimal siting of facilities. While 
recognising the importance of ground-level considerations when planning community health schemes for sustainability, we believe that the use of 'hard' OR techniques, as exemplified in Section 2, remains valid in ensuring efficiency of operation. We tailor our modelling to the particular situations of FBOs planning community health schemes in rural areas of developing countries. We give technical details of our range of models in (Smith et al., 2006).

Our modelling is designed for populations in rural areas: the effect of distances between settlements in such regions is to minimise inaccuracies of aggregation of demand. Types of road and conditions have an effect on accessibility, particularly in mountainous regions: we use weighted distances where appropriate for realism.

We take a hierarchical approach to modelling to be particularly appropriate, as health facilities are often provided at different levels, covering people resident within different distances. For example: a village health worker might provide a service to people living within a distance of, say, $2 \mathrm{~km}$, while community facilities might be utilised by people travelling from a radius of $7 \mathrm{~km}$ and hospitals from yet further afield. A referral system between different levels of provision is assumed to be necessary for the most effective use of facilities.

Differing poverty levels across villages of a target region effect planning for optimal location of facilities. Other factors, such as known disease incidence, may also be taken into consideration when siting services. We introduce an 'at-risk factor' in our models to account for such spatial variation, where appropriate.

Classical location analysis provides several models that are relevant to location of healthcare facilities. For example, as mentioned in several of the applications noted in Section 2, the $p$-median model (Hakimi, 1964, 1965) 
finds the optimal location of a given number of facilities to minimise the total distance travelled by people in the target area. An assumption is made that people travel to the nearest facility: we acknowledge the shortcomings of such a model, as pointed out by Yasenovskiy and Hodgson (2007) and Noor et al. (2006). Moreover, use of the p-median model can result in some people travelling excessive distances. However, we find that this type of model is useful in overall planning, giving a general picture of average distances travelled when services are essential to all those resident in a particular area. We therefore include $p$-median in our set of hierarchical models. In contrast, the maximal covering location model (Church and ReVelle, 1974), also used in several of the instances mentioned in Section 2, finds the maximum possible population that can be covered by a given number of facilities, by specifying the maximum desirable travel distance. We consider this type of model to be valid, particularly when considering non-essential, limited cover facilities, such as screening services. We have therefore made maximal cover location another part of our range of models.

We characterise the objectives that different service providers may make regarding access to hierarchical rural health facilities. Our contacts with health professionals working in different community health schemes in developing countries inform our modelling. For example, in some situations, it is desirable for access to hierarchical health services to be made always at the lowest levels. This is particularly the case when it is hoped to encourage first contact with a health systems through village health workers, rather than have people by-pass such services and go straight to community or hospital facilities. Alternatively, access to a healthcare system might be permitted at any level, as often happens in rural areas where customers can make a choice of facility. Where access is permitted at any level, a further differentiation occurs regarding the level at which services are provided. Certain types of service may be provided only at particular levels of a hierarchical system: cover at every level is then 
necessary, to enable all types of service to be available to all locations. On the other hand, one type of service, such as ante-natal care, might be provided in some form at all levels of a system, from rural village level to hospital. In such a care situation, the objective would be to cover as wide a spread of population as possible at some level.

Equitable access to health care is of importance to FBOs providing health care for those living at or below the poverty line. We therefore consider different equity objectives, or objective functions regarding equitable provision, as appropriate in our hierarchical models. We combine objectives for efficiency, i.e. p-median and maximal covering types, with objectives for equity. Our interpretation of equity of provision in the $p$-median, average distance, model is to minimise differences from a desired standard travel distance or time of travelling. In our maximal covering models we choose to minimise differences from a desired standard population covered per facility, as distances are automatically reduced to a given range with this form of objective function.

Our series of hierarchical models have been implemented and verified both in Visual $\mathrm{C}++$.NET and with the optimiser Xpress-MP. We summarise the different models as follows:

- Limited cover facilities: efficiency objective maximum cover type, with equity of population served.

1. HiMi-MCL-Eq: maximum cover by facilities at any level, with possible referral to higher levels.

2. HiMe-MCL-Eq: maximum cover at all levels.

3. HiS-MCL-Eq: maximum cover by level 1 (the lowest level) facilities, with possible referral.

- Essential facilities: efficiency objective p-median type, with equity of distance travelled. 
1. HiMi-PMP-Eq: total population-weighted distance travelled to nearest facility at any level, with referral.

2. HiMe-PMP-Eq: total population-weighted distance travelled to facilities at all levels.

3. HiS-PMP-Eq: total population-weighted distance travelled to a level 1 (lowest level) facility, with referral.

We illustrate our models with graphic output from HiMi-MCL-Eq and HiMe-MCL-Eq in Figures 1 and 2. Different emphases of our maximum cover models are shown, using data from a community health scheme run by Herbertpur Christian Hospital in Dehradun District, Uttaranchal, northern India. Figure 1 shows the pattern of lower level facilities stretching away from higher level ones, to maximise population covered at any possible level. In this case, it is assumed that services available at the lower levels of hierarchical services are also available at the higher levels. This modelling is useful in situations of sparse cover of facilities, for example in rural areas. Figure 2 shows a situation where all locations must ideally be covered at all levels. Cover is therefore available for only a portion of the population of the area. Such modelling is suited to districts that are relatively well supplied with facilities.

\section{A case study: the REACH scheme, modelling sustainable facilities}

Healthcare financing is much under consideration by organisations such as EHA, for those without ready access to state-provided quality health care. Health insurance schemes exist in many forms, often aimed at individual cover. However, for those living in near poverty conditions, there is little incentive to spend income on reduction of possible future misfortune caused by illness, when day-to-day concerns are more pressing. 


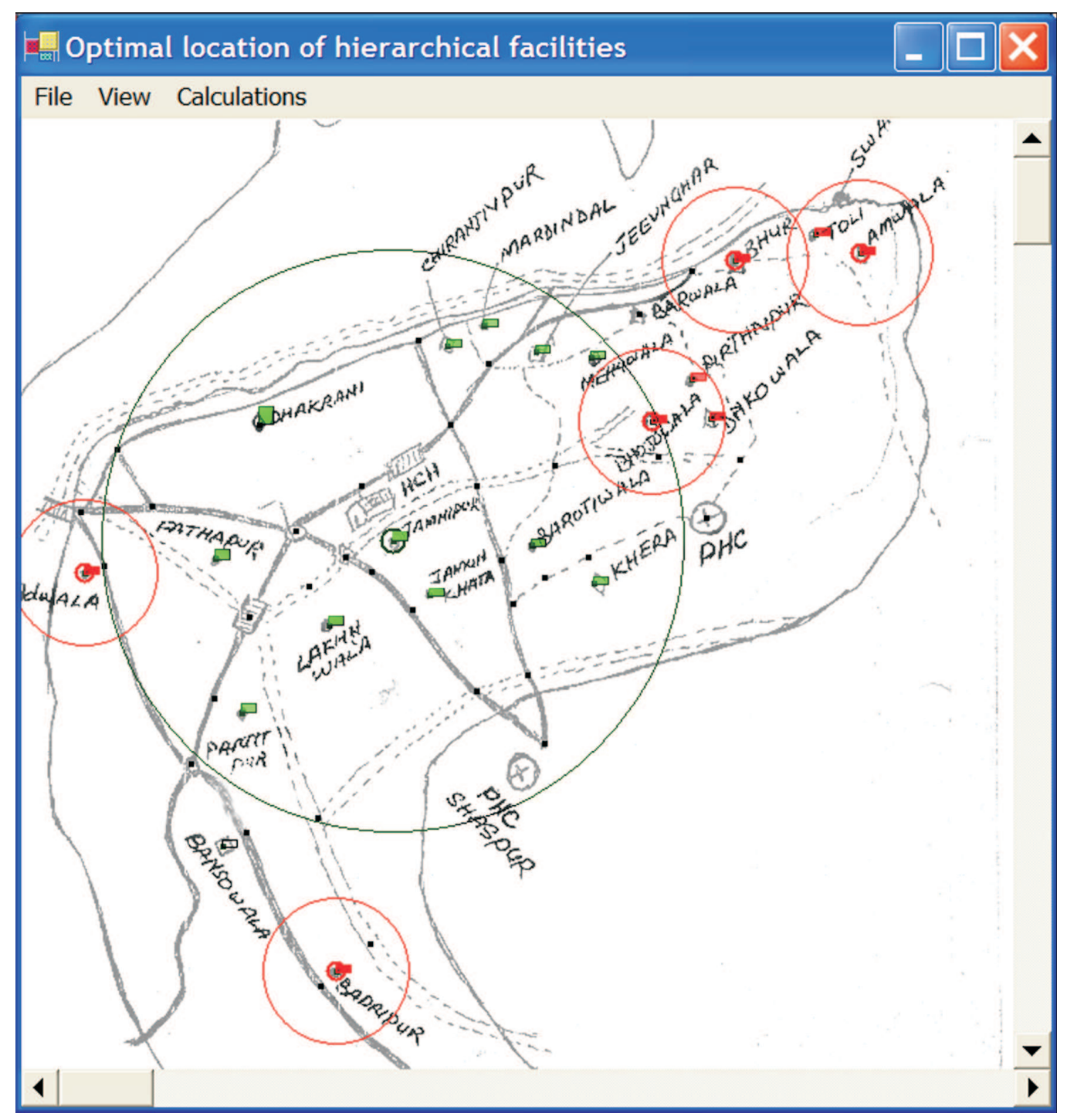

Figure 1: Location of 5 lower level facilities and 1 higher level facility to maximise population covered at any possible level. Population sizes are indicated by rectangles at different village locations. Smaller circles represent village level facilities; the larger circle shows the higher level of cover. 




Figure 2: Location of 4 lowest level facilities, 2 middle and 1 highest level facility, to maximise population covered by every level of hierarchical facilities. Population sizes are indicated by rectangles at different village locations; unshaded rectangles show villages not covered at every level. Size of circles gives the size of cover by facilities at different levels. 
Community health insurance may be a more attractive proposition, especially if coupled with a readily-available local service. Furthermore, re-insurance of the most serious health needs at national level may be coupled with local schemes to give comprehensive cover. Feeley et al. (2002) evaluate such a project introduced in the Phillippines.

A project to be piloted in South India by Transformational Business Network, the REACH scheme (Morse, 2006), aims to offer a sustainable primary healthcare service to people living in rural areas, financed through existing women's self-help groups (SHGs). Those participating in SHGs make very small regular savings, which can be used to take out micro-finance loans for starting small businesses. The loans are then paid back as profits are reaped. In the REACH scheme, it is hoped that, alongside saving for future loans, it will be attractive for group members to invest an additional small amount in a primary healthcare scheme with evident benefits. HCWs will be employed by REACH in regions where there is a sufficient density of SHGs to maintain their employment. The HCWs will travel around villages within reach, offering a basic health service to members of SHGs and their families, while raising extra income from non-SHG members.

A number of questions may be posed concerning the planning of such schemes. For example, 'What is the minimum number of HCWs needed to cover any particular region?' Modelling on the lines of the location set covering problem (Toregas et al., 1971), could answer this question. Also, 'What is the maximum sustainable number of HCWs that can operate in a particular region, given the geography and existence of SHGs? At which locations should the HCWs be based?' We model this situation with a new covering-type model, that maximises the number of health workers who can work sustainably in an area, given a sufficient number of SHGs within a certain travel distance of the HCW bases. Details are given below. Though designed for a particular situation, this model could be used in general to 
locate a maximal number of sustainable facilities. However, it should be noted that set-up and operating costs in the REACH project are calculated to be sustainably covered by ongoing revenues, given sufficient volume of demand. Thus high fixed set-up costs do not constrain the number of HCWs, as commonly occurs in other applications.

The modelling involves a number of simplifications. As with the hierarchical models described in Section 4, we consider loss of accuracy due to aggregation of demand at village centres to be minimal because of distances between rural communities. A number of SHGs are assumed to be present at each village location. Another simplification is that we do not allow for the routes taken by an HCW in each day's travel; future research could be undertaken into such considerations. Additionally, our model could be expanded to take referrals to higher levels of hierarchical services into account.

\subsection{MNS Model for the location of the maximal number of sustainable facilities}

We introduce the MNS model for the location of the maximal number of sustainable facilities. Implementation and verification has taken place in both Visual C++.NET and the optimiser Xpress-MP.

We maximise the sustainable number of HCWs, with a sufficient number, $G$, of self-help groups allocated to each HCW within a given travel distance, $D$, of their home bases (or facility locations). Demand is situated at demand nodes $i(i \in I$, the set of demand nodes). HCWs may be located at potential facility nodes $j(j \in J$, the set of candidate facility nodes).

\section{Notation:}

$s_{i}=$ number of self-help groups at demand node $i, i \in I$. 
$d_{i j}=$ distance between node $i$ and node $j, i \in I, j \in J$.

$c_{i j}=\left\{\begin{array}{l}1 \text { if } d_{i j} \leq D, \\ 0 \text { otherwise, }\end{array} \quad i \in I, j \in J\right.$.

$p_{j}=\left\{\begin{array}{l}1 \text { if a HCW pre-exists at node } j, \quad j \in J . \\ 0 \text { otherwise, }\end{array}\right.$

Decision variables are:

$$
\begin{aligned}
& X_{j}= \begin{cases}1 & \text { if an HCW is located at node } j, j \in J . \\
0 & \text { otherwise, }\end{cases} \\
& Y_{i j}= \begin{cases}1 & \text { if demand at node } i \text { is allocated to an HCW at node } j, i \in I, j \in J . \\
0 & \text { otherwise, }\end{cases}
\end{aligned}
$$

Maximise

$$
Z=\sum_{j \in J} X_{j}
$$

subject to

$$
\begin{aligned}
& \sum_{j \in J} Y_{i j} \leq 1, \quad i \in I, \\
& Y_{i j} \leq c_{i j} X_{j}, \quad i \in I, j \in J, \\
& \sum_{i \in I} s_{i} Y_{i j} \geq X_{j} G, \quad j \in J, \\
& X_{j}+Y_{i l} \leq 1, \quad i \in I, j \in J, l \in J \mid d_{i j}<d_{i l}, \\
& X_{j} \geq p_{j} \quad j \in J .
\end{aligned}
$$

Demand from each village is allocated to at most $1 \mathrm{HCW}$ via constraint (2). Constraint (3) ensures that for demand to be covered, it must be sufficiently close to an open facility, i.e. to an HCW base. Constraint (4) specifies the minimum number of self-help groups that an HCW must cover for sustainability. Constraint (6) enables pre-located HCWs to be specified. 
Demand is allocated to the nearest HCW by constraint (5). The constraint is concerned with conditions where $d_{i j}<d_{i l}$, i.e. demand node $i$ is nearer to facility node $j$ than node $l$. Given those conditions, if a facility is open at node $j$, demand may not be allocated to a facility at node $l$. We draw attention to the linear nature of this minimum distance constraint, which we report gives ease of solution and verification using the optimiser Xpress-MP. We use similar constraints to achieve allocation to nearest facilities in our hierarchical models (Smith et al., 2006).

\subsection{Illustrative model results}

Figure 3 shows output from the MNS model, using illustrative data for numbers of SHGs at different village centres. A maximum of 5 sustainable facilities can be opened (i.e. $5 \mathrm{HCWs}$ can be employed sustainably), if an $\mathrm{HCW}$ can travel within a radius of $2.6 \mathrm{~km}$ and must cover a minimum of 8 SHGs.

To illustrate the model functionality, we demonstrate in Table 1 the effects of changing the parameters of distance covered and minimum number of SHGs needed for sustainability. It can be observed that sensitivity to distance travelled lessens with the minimum number of SHGs to be covered. Such information gives local decision-makers a range of options when deciding upon location of HCWs, given travel possibilities and SHG numbers.

\section{Conclusion and suggestions for future work}

We present a number of hierarchical location models uniquely aimed at efficient and equitable planning of community health schemes. We consider the importance of ground-level factors in achieving sustainability for any project, and claim that there is an equally valid place for analytical modelling in planning such ventures. Our contacts with healthcare 


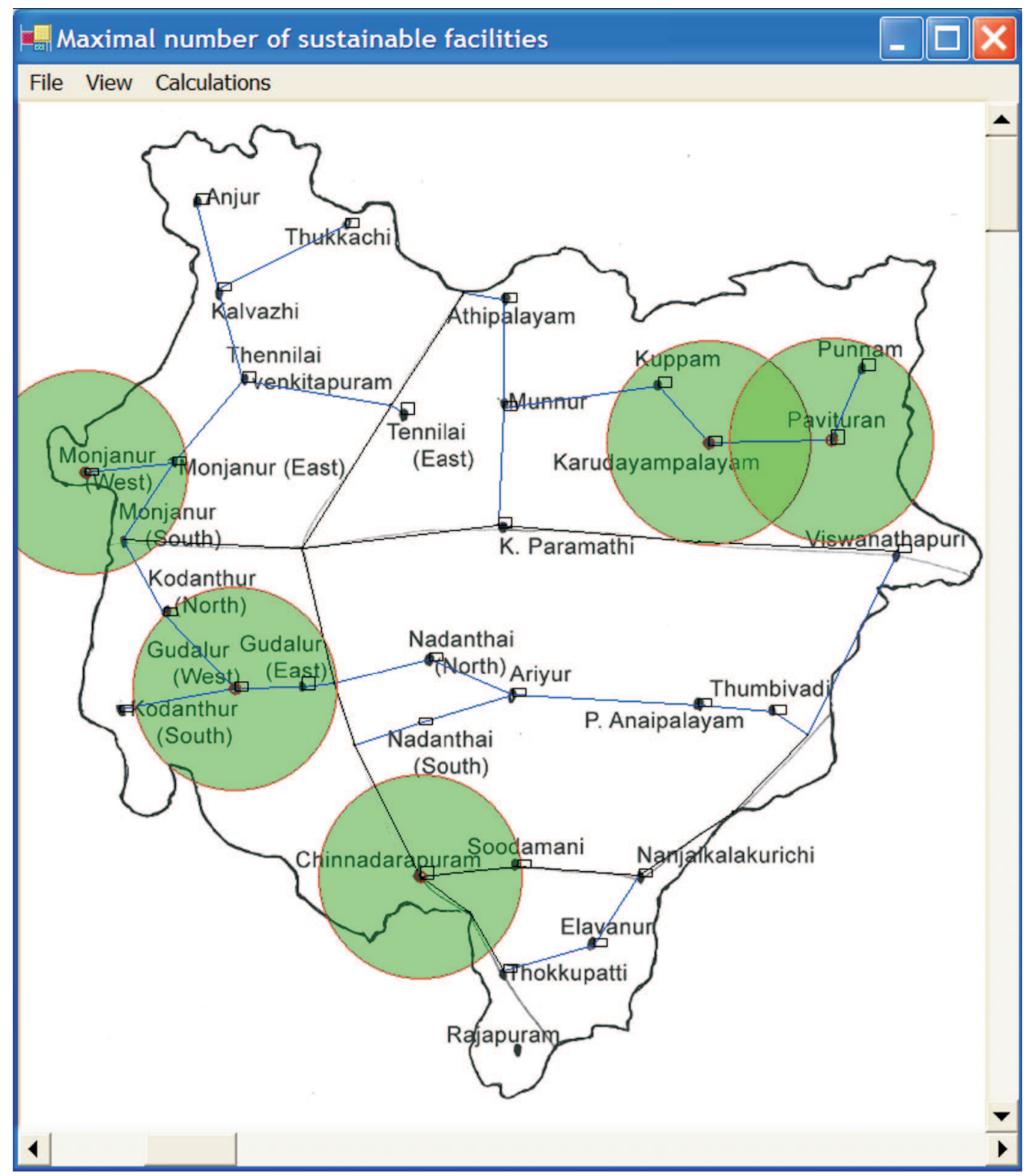

Figure 3: MNS output: location of the maximal number of sustainable facilities in the Aravakurichi Taluk of Karur District, Tamil Nadu, southern India. Shaded circles represent areas where HCWs can be sustainably employed. Relative numbers of SHGs in each village are shown by unshaded rectangles. 


\begin{tabular}{c|cccccc}
\hline $\begin{array}{c}\text { Distance, } \boldsymbol{D} \\
(\mathbf{k m})\end{array}$ & \multicolumn{7}{|c}{$\boldsymbol{G}$} \\
\cline { 2 - 7 } $\mathbf{5}$ & $\mathbf{6}$ & $\mathbf{7}$ & $\mathbf{8}$ & $\mathbf{9}$ & $\mathbf{1 0}$ \\
\hline 1.0 & 2 & 0 & 0 & 0 & 0 & 0 \\
1.1 & 3 & 1 & 0 & 0 & 0 & 0 \\
1.2 & 3 & 1 & 0 & 0 & 0 & 0 \\
1.3 & 4 & 2 & 1 & 1 & 1 & 0 \\
1.4 & 4 & 2 & 1 & 1 & 1 & 0 \\
1.5 & 4 & 2 & 1 & 1 & 1 & 0 \\
1.6 & 5 & 4 & 1 & 1 & 1 & 0 \\
1.7 & 6 & 4 & 2 & 1 & 1 & 0 \\
1.8 & 6 & 5 & 4 & 2 & 1 & 0 \\
1.9 & 6 & 5 & 4 & 2 & 1 & 0 \\
2.0 & 6 & 5 & 4 & 3 & 3 & 1 \\
2.1 & 6 & 5 & 4 & 3 & 3 & 1 \\
2.2 & 6 & 5 & 4 & 3 & 3 & 1 \\
2.3 & 6 & 5 & 4 & 3 & 3 & 1 \\
2.4 & 6 & 5 & 5 & 4 & 3 & 1 \\
2.5 & 6 & 5 & 5 & 4 & 3 & 2 \\
2.6 & 6 & 5 & 5 & 5 & 3 & 3 \\
\hline
\end{tabular}

Table 1: Maximal numbers of sustainable facilities: variation with distance and numbers of SHGs that must be covered for sustainability. 
professionals in the field of community health give credence to this view. Providers of community healthcare programmes can benefit from the use of OR modelling to improve planning in several contexts: a) when communicating with funding bodies, b) when planning day-to-day operations, and c) in explaining decision-making at village level. However, as Rahman and Smith (2000) point out, implementation of OR solutions requires close collaboration between modellers, decision makers and, in this case, medical specialists. Communication problems abound, and it is helpful to have the involvement of people who can speak the languages of both community health practice and operational research technicalities.

In addressing sustainable development directly, we propose the MNS model for the location of the maximal number of sustainable facilities. This model is designed for the situation of the REACH project Morse (2006), in seeking to establish sustainable employment of healthcare workers offering services via self-help groups in village locations. Possible extensions to this modelling are proposed.

Our future research is directed towards the modelling of demand for health facilities in rural regions of developing countries. Variability of demand affects the sustainability of self-funding projects. Also of interest is the growth of use of facilities in time as social developments mature, since our experience of community health projects convinces us that their sustainability is closely linked with growth in economic development.

\section{Acknowledgements}

This research is funded by the EPSRC (DTA grant EP/P500478/1). Xpress-MP is made available by Dash Optimization's Academic Partnership Program.

We acknowledge the helpful comments of the anonymous referees. 
We are grateful for the advice and support of: staff of Herbertpur Christian Hospital, Uttaranchal, India and of Prem Sewa Hospital, Utraula, Uttar Pradesh, India; Mr M. Morse of Transformational Business Network, Healthcare Resource Development Group; Dr T. Lankester, Community Health Global Network; Dr N. Grills, community health physician; and Dr G. Barber, midwifery consultant. 


\section{References}

Banerji, S. H., Fisher, H. B., 1974. Hierarchical location analysis for integrated area planning in rural India. Papers of the Regional Science Association 33, 177-194.

Barr, N., 2002. Foreword. In: Dror, D. M., Preker, A. S. (Eds.), Social Reinsurance: a new approach to sustainable community health financing. The World Bank and The International Labour Office, Washington, D.C. and Geneva, pp. xiii-xiv.

Church, R. L., ReVelle, C., 1974. The maximal covering location problem. Papers of the Regional Science Association 32, 101-118.

Eaton, D. J., Church, R. L., Bennett, V. L., Hamon, B. L., 1981. On deployment of health resources in rural Valle del Cauca, Colombia. TIMS Studies in the Management Sciences 17, 331-359.

EHA, 2006. Emmanuel Hospital Association. Community Health and Development Projects. Viewed online on 19/09/2006 at http://www.ehahealth.org/community.htm.

Feeley, F. G., Gasparro, D. J., Snowden, K., 2002. Assessment of piloting social reinsurance in the philippines. In: Dror, D. M., Preker, A. S. (Eds.), Social Reinsurance: a new approach to sustainable community health financing. The World Bank and The International Labour Office, Washington, D.C. and Geneva, pp. 424-446.

Hakimi, S. L., 1964. Optimum locations of switching centers and the absolute centers and medians of a graph. Operations Research 12, 450-459.

Hakimi, S. L., 1965. Optimum distribution of switching centers in a communication network and some related graph theoretic problems. Operations Research 13, 462-475. 
Hodgson, M. J., 1988. An hierarchical location-allocation model for primary health care delivery in a developing area. Social Science and Medicine 26 (1), 153-161.

Hodgson, M. J., Laporte, G., Semet, F., 1998. A covering tour model for planning mobile health care facilities in Suhum District, Ghana. Journal of Regional Science 38 (4), 621-638.

Jackson, J., 2006. Reaching the hard to reach - the challenges of aa community operational researcher. OR Insight 19, 193-209.

Källander, K., Tomson, G., Nsabagasani, X., Sabiti, J. N., Pariyo, G., Peterson, S., 2006. Can community health workers recognize pneumonia in children? Experiences from western Uganda. Transactions of the Royal Society of Tropical Medicine and Hygiene 100, 956-963.

Lankester, T. E., 2000. Setting up community health programmes. Macmillan Education, Oxford.

Lewis, H., Rudolph, M., White, L., 2003. Rapid appraisal of the health promotion needs of the Hillbrow Community, South Africa. International Journal of Healthcare Technology and Management 5, 20-33.

Mingers, J., Rosenhead, J., 2001. Diverse unity; looking inward and outward. In: Rosenhead, J., Mingers, J. (Eds.), Rational analysis for a problematic world revisited. John Wiley and Sons, Ltd., Chichester, pp. 337-355.

Morse, M., 2006. REACH: Responsible, Empowering, Accessible Community Healthcare, Transformational Business Network, Healthcare Resource Development Group.

Noor, A. M., Amin, A. A., Gething, P. W., Atkinson, P. M., Hay, S. I., Snow, R. W., 2006. Modelling distances travelled to government health services in Kenya. Tropical Medicine and International Health 11, 188-196. 
Patel, N. R., 1979. Locating rural social service centers in India. Management Science 25, 22-30.

Preker, A. S., Langenbrunner, J., Jakab, M., 2002. Rich-poor differences in health financing. In: Dror, D. M., Preker, A. S. (Eds.), Social Reinsurance: a new approach to sustainable community health financing. The World Bank and The International Labour Office, Washington, D.C. and Geneva, pp. 21-36.

Rahman, S., Smith, D. K., 2000. Use of location-allocation models in health service development planning in developing nations. European Journal of Operational Research 123 (3), 437-452.

Rushton, G., 1984. Use of location-allocation models for improving the geographical accessibility of rural services in developing countries. International Regional Science Review 9 (3), 217-240.

Smith, H. K., Harper, P. R., Potts, C. N., 2006. Bicriteria efficiency/equity hierarchical location models for application in healthcare and other sectors, in preparation.

Toregas, C., Swain, R., Revelle, C., Bergman, L., 1971. The location of emergency service facilities. Operations Research 19, 1363-1373.

UN, 2002. The Millennium Development Goals and the United Nations Role. United Nations Department of Public Information.

UN, 2006. The Millennium Development Goals Report. United Nations Department of Economic and Social Affairs, DESA.

WHO, 2002. Innovative care for chronic conditions; building blocks for action. World Health Organization, Geneva.

Yasenovskiy, V. S., Hodgson, M. J., 2007. Hierarchical location-allocation with spatial choice interaction modeling. Annals of the Association of American Geographers. Article in press. 